\title{
The Hilbert Curve of a 4-dimensional Scroll with a Divisorial Fiber
}

\author{
Antonio Lanteri* and Andrea Luigi Tironi
}

\begin{abstract}
In dimension $n=2 m-2 \geq 4$ adjunction theoretic scrolls over a smooth $m$-fold may not be classical scrolls, due to the existence of divisorial fibers. A 4dimensional scroll $(X, L)$ over $\mathbb{P}^{3}$ of this type is considered, and the equation of its Hilbert curve $\Gamma$ is determined in two ways, one of which relies on the fact that $(X, L)$ is at the same time a classical scroll over a threefold $Y \neq \mathbb{P}^{3}$. It turns out that $\Gamma$ does not perceive divisorial fibers. The equation we obtain also shows that a question raised in 2] has negative answer in general for non-classical scrolls over a 3-fold. More precisely, the answer for $(X, L)$ is negative or positive according to whether $(X, L)$ is regarded as an adjunction theoretic scroll or as a classical scroll; in other words, it is the answer to this question to distinguish between the existence of jumping fibers or not.
\end{abstract}

\section{Introduction}

For a polarized manifold $(\mathcal{X}, \mathcal{L})$ of dimension $n$, two notions of scroll over a variety $\mathcal{Y}$ of smaller dimension $m$ are possible: $(\mathcal{X}, \mathcal{L})$ is a classical scroll if $\mathcal{X}=\mathbb{P}(\mathcal{E})$ for an ample vector bundle $\mathcal{E}$ on $\mathcal{Y}, \mathcal{L}$ being the tautological line bundle, while $(\mathcal{X}, \mathcal{L})$ is an adjunction theoretic scroll over $\mathcal{Y}$ if there exists a surjective morphism $\varphi: \mathcal{X} \rightarrow \mathcal{Y}$ such that $K_{\mathcal{X}}+(n-m+1) \mathcal{L}=\varphi^{*} \mathcal{A}$ for some ample line bundle $\mathcal{A}$ on $\mathcal{Y}$ (see [4, p. 81]). Essentially, classical scrolls are also adjunction theoretic scrolls, by taking as $\varphi$ the bundle projection $p: \mathcal{X} \rightarrow \mathcal{Y}$, except when $K_{\mathcal{Y}}+\operatorname{det} \mathcal{E}$ fails to be ample, and all these exceptions are well known in low dimension (see, [3], [8, §3] and [9, §4.2]). Conversely, it is known that for $m \leq 4$ an adjunction theoretic scroll is a classical scroll if $n \geq 2 m-1$, when $\mathcal{L}$ is very ample (see [4, Proposition 14.1.3] and [8, Theorem 2.2]). This is no longer true for $n=2 m-2 \geq 4$, since in this case $\varphi$ can admit divisorial fibers. A class of examples illustrating this phenomenon is due to Beltrametti and Sommese [3, (4.2)].

In this paper, a 4 -dimensional scroll $(X, L)$ over $\mathbb{P}^{3}$ - the simplest example of this type - is considered and the equation of its Hilbert curve is determined. This is done in two different ways: the former is via the explicit Riemann-Roch formula for 4-folds

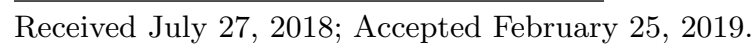

Communicated by Yoichi Miyaoka.

2010 Mathematics Subject Classification. Primary: 14C20, 14N30; Secondary: 14J35, 14 M99.

Key words and phrases. scroll, divisorial fiber, Hilbert curve.

*Corresponding author. 
exploiting that $(X, L)$ itself is also a classical scroll over another threefold $Y$, related to $\mathbb{P}^{3}$ (Section 3); the latter relies on a recursive procedure introduced in [7, Section 4], working for scrolls of both types (Section 4). It turns out that the Hilbert curve does not detect divisorial fibers. Moreover, the equation we obtain indicates that a question raised in [2] has negative answer in general for non-classical scrolls. More precisely, it turns out that for our $(X, L)$ the answer is negative or positive according to whether we look at it either as an adjunction theoretic scroll over $\mathbb{P}^{3}$, or as a classical scroll over $Y$; in other words, it is the answer to this question to distinguish between the existence of jumping fibers or not.

\section{Preliminaries}

Varieties considered in this paper are defined over the field $\mathbb{C}$ of complex numbers. We use the standard notation and terminology from algebraic geometry. A manifold is any smooth projective variety. Tensor products of line bundles are denoted additively. The pullback of a vector bundle $\mathcal{E}$ on a manifold $\mathcal{X}$ by an embedding $\mathcal{Z} \rightarrow \mathcal{X}$ is simply denoted by $\mathcal{E}_{\mathcal{Z}}$, while $K_{\mathcal{X}}$ will stand for the canonical bundle of $\mathcal{X}$. A polarized manifold is a pair $(\mathcal{X}, \mathcal{L})$ consisting of a manifold $\mathcal{X}$ and an ample line bundle $\mathcal{L}$ on $\mathcal{X}$.

For the notion and the general properties of the Hilbert curve associated to a polarized manifold we refer to [2], see also [6]. Here we just recall some basic facts. Let $(\mathcal{X}, \mathcal{L})$ be a polarized manifold of dimension $n \geq 2$ and regard $\mathrm{N}(\mathcal{X}):=\operatorname{Num}(\mathcal{X}) \otimes_{\mathbb{Z}} \mathbb{C}$ as a complex affine space. If $\operatorname{rk}\left\langle K_{\mathcal{X}}, \mathcal{L}\right\rangle=2$, we can consider the plane $\mathbb{A}^{2}=\mathbb{C}\left\langle K_{\mathcal{X}}, \mathcal{L}\right\rangle \subset \mathrm{N}(\mathcal{X})$, generated by the classes of $K_{\mathcal{X}}$ and $\mathcal{L}$. For any line bundle $D$ on $\mathcal{X}$ the Riemann-Roch theorem provides an expression for the Euler-Poincaré characteristic $\chi(D)$ in terms of $D$ and the Chern classes of $\mathcal{X}$. Let $p$ denote the complexified polynomial of $\chi(D)$, when we set $D=x K_{\mathcal{X}}+y \mathcal{L}$, with $x, y$ complex numbers, namely $p(x, y)=\chi\left(x K_{\mathcal{X}}+y \mathcal{L}\right)$. The Hilbert curve of $(\mathcal{X}, \mathcal{L})$ is the complex affine plane curve $\Gamma=\Gamma_{(\mathcal{X}, \mathcal{L})} \subset \mathbb{A}^{2}$ of degree $n$ defined by $p(x, y)=0$ [2, Section 2]. Notice that the Hilbert curve can be defined also when the numerical classes of $K_{\mathcal{X}}$ and $\mathcal{L}$ are linearly dependent, but in this case, the $(x, y)$-plane is only formal, $\Gamma_{(\mathcal{X}, \mathcal{L})}$ losing the meaning of a plane section of the Hilbert variety of $\mathcal{X}$ (see [2, Section 2]). For example, the Hilbert curve of $\left(\mathbb{P}^{n}, \mathcal{O}_{\mathbb{P}^{n}}(r)\right)$ has the following equation (see, e.g., [2, p. 465] and [7, Theorem 2.7]):

$$
p(x, y)=\frac{(-1)^{n}}{n !} \prod_{i=1}^{n}((n+1) x-r y-i) .
$$

Due to Serre duality, $\Gamma$ is invariant under the involution $D \mapsto K_{\mathcal{X}}-D$ acting on $\mathrm{N}(\mathcal{X})$. Sometimes, to make this symmetry more evident, it is convenient to represent $\Gamma$ in terms of the affine coordinates $(u=x-1 / 2, v=y)$ rather than $(x, y)$. So, rewriting our divisor 
as $D=\frac{1}{2} K_{\mathcal{X}}+\Delta$, where $\Delta=u K_{\mathcal{X}}+v \mathcal{L}, \Gamma$ can be represented with respect to these coordinates by $p(1 / 2+u, v)=0$. We refer to this equation as the canonical equation of $\Gamma$. It is immediate to check that any nontrivial homogeneous part in the corresponding polynomial in $u, v$ has degree with the same parity as $n$; for instance, on a smooth 4 -fold $\mathcal{X}$, for any divisor $D=\frac{1}{2} K_{\mathcal{X}}+\Delta$ the Riemann-Roch formula gives

$$
\chi(D)=\frac{1}{24} \Delta^{4}+\frac{1}{48}\left(2 c_{2}(\mathcal{X})-K_{\mathcal{X}}^{2}\right) \cdot \Delta^{2}+\frac{1}{384}\left(K_{\mathcal{X}}^{2}-4 c_{2}(\mathcal{X})\right) \cdot K_{\mathcal{X}}^{2}+\chi\left(\mathcal{O}_{\mathcal{X}}\right)
$$

(e.g., see [1, p. 292]). We thus see that for a polarized 4-fold, the polynomial $p$ contains only homogeneous parts of degree 4 and 2 in $u, v$ plus the constant term: so, if the latter is zero, then $\Gamma$ has a singular point at the origin.

The most significant property of the Hilbert curve of $(\mathcal{X}, \mathcal{L})$ is its sensitivity with respect to fibrations that suitable adjoint linear systems to $\mathcal{L}$ may induce on $\mathcal{X}[2$, Theorem 6.1]. This makes scrolls (of any type) very interesting from the point of view of their Hilbert curves. In fact if $(\mathcal{X}, \mathcal{L})$ is a scroll over $\mathcal{Y}$, with $\operatorname{dim} \mathcal{Y}=m$, then $\Gamma_{(\mathcal{X}, \mathcal{L})}$ consists of $n-m$ parallel lines plus a curve $C$, of degree $m$, and we can consider the following question (see [2, Problem 6.6]).

Question 2.1. Can $C$ itself be regarded as the Hilbert curve of $\mathcal{Y}$, polarized by some ample $\mathbb{Q}$-line bundle?

For instance, for scrolls over a smooth curve the answer is positive [6, Remark 4.1]. This note is mainly concerned with the answer to Question 2.1 for the 4-scroll $(X, L)$ described below (see also [4, p. 330], [3]). Set $X=\mathbb{P}_{Y}(\mathcal{F})$ and let $p: X \rightarrow Y$ be the projection, where $Y$ is $\mathbb{P}^{3}$ blown-up at a point $w, \mathcal{F}=H^{\oplus 2}$, and $H=\sigma^{*} \mathcal{O}_{\mathbb{P}^{3}}(3)-e$, $\sigma: Y \rightarrow \mathbb{P}^{3}$ standing for the blowing-up and $e \cong \mathbb{P}^{2}$ for the exceptional divisor. We denote by $L$ the tautological line bundle of $\mathcal{F}$ on $X$. Clearly, $(X, L)$ is a classical scroll over $Y$ via $p$, while it is an adjunction theoretic scroll over $\mathbb{P}^{3}$ via the map $\pi:=\sigma \circ p: X \rightarrow \mathbb{P}^{3}$, since

$$
K_{X}+2 L=p^{*}\left(K_{Y}+2 H\right)=\pi^{*}\left(K_{\mathbb{P}^{3}}+2 \mathcal{O}_{\mathbb{P}^{3}}(3)\right)=\pi^{*} \mathcal{O}_{\mathbb{P}^{3}}(2) .
$$

However, it is not a classical scroll over $\mathbb{P}^{3}$, since the fiber $\pi^{-1}(w)=\mathbb{P}_{e}\left(\mathcal{F}_{e}\right)$ is a divisor inside $X$, being isomorphic to $e \times \mathbb{P}^{1}$. The following diagram summarizes the above situation

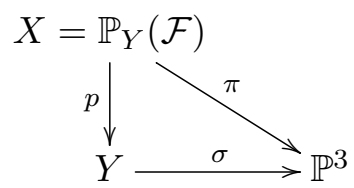

Before addressing Question 2.1 for $(X, L)$, we need the equation of $\Gamma_{(X, L)}$. 
Proposition 2.2. Let $(X, L)$ be the pair described above. The canonical equation of $\Gamma_{(X, L)}$ in coordinates $(u, v)$, is

$$
p_{(X, L)}\left(\frac{1}{2}+u, v\right)=\frac{1}{3}(2 u-v)(u-v)\left(28 u^{2}-38 u v+13 v^{2}-1\right)=0 .
$$

Sections 3 and 4 contain two different proofs of this statement.

\section{First approach}

Here, to get the canonical equation of the Hilbert curve $\Gamma_{(X, L)}$ we implement 2.2 with $\mathcal{X}=X$ and $\Delta=u K_{X}+v L$.

First of all we recall the Chern-Wu relation:

$$
L^{2}-L \cdot p^{*} c_{1}(\mathcal{F})+p^{*} c_{2}(\mathcal{F})=0
$$

Since $\mathcal{F}=H^{\oplus 2}$, it gives $L^{2}=L \cdot p^{*}(2 H)-p^{*}\left(H^{2}\right)$. Moreover, for any divisor $D$ on $Y$, we get

$$
L \cdot p^{*} D^{3}=D^{3}, \quad L^{2} \cdot p^{*} D^{2}=2 H \cdot D^{2}, \quad L^{3} \cdot p^{*} D=3 H^{2} \cdot D,
$$

and $L^{4}=4 H^{3}$. Let $h=\sigma^{*} \mathcal{O}_{\mathbb{P}^{3}}(1)$; then $H=3 h-e$, hence $H^{3}=26$, since $h^{3}=$ $\left(\mathcal{O}_{\mathbb{P}^{3}}(1)\right)^{3}=1, e^{3}=\left(\mathcal{O}_{e}(e)\right)^{2}=1$, and $h \cdot e=0$. Therefore

$$
L^{4}=104
$$

Moreover, specializing the above intersections for $D=h$ and $D=e$ respectively, we get

$$
\begin{aligned}
& L \cdot p^{*} h^{3}=1, \quad L^{2} \cdot p^{*} h^{2}=6, \quad L^{3} \cdot p^{*} h=27, \\
& L \cdot p^{*} e^{3}=1, \quad L^{2} \cdot p^{*} e^{2}=-2, \quad L^{3} \cdot p^{*} e=3 .
\end{aligned}
$$

Now look at the Chern classes of $X$. We have $K_{X}=-2 L+p^{*}\left(K_{Y}+2 H\right)$, by the canonical bundle formula for $X$, since $\mathcal{F}=H^{\oplus 2}$. Moreover, since $K_{Y}=-4 h+2 e$, we get $K_{Y}+2 H=2 h$, hence

$$
K_{X}=-2 L+p^{*}\left(K_{Y}+2 H\right)=-2\left(L-p^{*} h\right) .
$$

Consequently,

$$
\begin{gathered}
K_{X}^{2}=4\left(L^{2}-2 L \cdot p^{*} h+p^{*} h^{2}\right), \\
K_{X}^{3}=-8\left(L^{3}-3 L^{2} \cdot p^{*} h+3 L \cdot p^{*} H^{2}-p^{*} h^{3}\right),
\end{gathered}
$$

and

$$
K_{X}^{4}=16\left(L^{4}-4 L^{3} \cdot p^{*} h+6 L^{2} \cdot p^{*} h^{2}-4 L \cdot p^{*} h^{3}\right)=16 \times 28=448
$$


Combining these with 3.2 we can compute the pluridegrees $K_{X}^{i} \cdot L^{4-i}$ for $i=1,2,3$ :

$$
K_{X} \cdot L^{3}=-154, \quad K_{X}^{2} \cdot L^{2}=224, \quad K_{X}^{3} \cdot L=-320 .
$$

This provides the values of several intersection products in the Riemann-Roch formula, but many other involve the second Chern class of $X$. To evaluate it, looking at the $\mathbb{P}^{1}$-bundle structure $p: X \rightarrow Y$, we can use the relative tangent sequence

$$
0 \rightarrow T_{X / Y} \rightarrow T_{X} \rightarrow p^{*} T_{Y} \rightarrow 0
$$

and the relative Euler sequence

$$
0 \rightarrow \mathcal{O}_{X} \rightarrow p^{*} \mathcal{F}^{\vee} \otimes L \rightarrow T_{X / Y} \rightarrow 0
$$

Combining them, we get the following relation between the Chern polynomials

$$
c\left(T_{X} ; t\right)=p^{*} c\left(T_{Y} ; t\right) c\left(p^{*} \mathcal{F}^{\vee} \otimes L ; t\right),
$$

which gives

$$
c_{2}(X)=p^{*} c_{2}(Y)+p^{*} c_{1}(Y) \cdot c_{1}\left(p^{*} \mathcal{F}^{\vee} \otimes L\right)+c_{2}\left(p^{*} \mathcal{F}^{\vee} \otimes L\right) .
$$

Recall that $c_{2}(Y)=\sigma^{*} c_{2}\left(\mathbb{P}^{3}\right)$ (e.g., see $\left[5\right.$, Lemma at p. 609]), hence $c_{2}(Y)=6 h^{2}$. Moreover, $c_{1}\left(p^{*} \mathcal{F}^{\vee} \otimes L\right)=2\left(L-p^{*} H\right)$ and $c_{2}\left(p^{*} \mathcal{F}^{\vee} \otimes L\right)=\left(L-p^{*} H\right)^{2}$. So, taking into account the expressions of $H$ and $K_{Y}$ in terms of $h$ and $e$, we obtain

$$
c_{2}(X)=L^{2}+2 L \cdot p^{*}(h-e)-3 p^{*}\left(3 h^{2}+e^{2}\right) .
$$

This gives

$$
c_{2}(X) \cdot K_{X}^{2}=4\left(L^{4}-2 L^{3} \cdot p^{*} e-3 L^{2} \cdot p^{*}\left(4 h^{2}+e^{2}\right)+20 L \cdot p^{*} h^{3}\right)=4 \times 52=208 .
$$

Moreover,

$$
2 c_{2}(X)-K_{X}^{2}=-2\left(L^{2}-2 L \cdot p^{*}(3 h-e)+p^{*}\left(11 h^{2}+3 e^{2}\right)\right) .
$$

As a consequence of the above relations we get

$$
\left(2 c_{2}(X)-K_{X}^{2}\right) \cdot K_{X}^{2}=-32,\left(2 c_{2}(X)-K_{X}^{2}\right) \cdot K_{X} \cdot L=24,\left(2 c_{2}(X)-K_{X}^{2}\right) \cdot L^{2}=-16
$$

Now we have all ingredients; so, letting $\Delta=u K_{X}+v L, 2.2$ allows us to express the canonical equation of the Hilbert curve $\Gamma_{(X, L)}$. First of all, since $\chi\left(\mathcal{O}_{X}\right)=1$, from (3.3) and $(3.5)$ we get the degree zero term, which is

$$
\frac{1}{384}\left(K_{X}^{2}-4 c_{2}(X)\right) \cdot K_{X}^{2}+\chi\left(\mathcal{O}_{X}\right)=\frac{1}{384}(448-832)+1=0 .
$$


This means that $\Gamma_{(X, L)}$ has a singular point of multiplicity $\geq 2$ at the origin. Next, since

$$
\begin{aligned}
\left(2 c_{2}(X)-K_{X}^{2}\right) \cdot \Delta^{2} & =-32 u^{2}+48 u v-16 v^{2}=-16\left(2 u^{2}-3 u v+v^{2}\right) \\
& =-16(2 u-v)(u-v),
\end{aligned}
$$

in view of the previous computations, the homogeneous part of degree 2 is

$$
\frac{1}{48}\left(2 c_{2}(X)-K_{X}^{2}\right) \cdot \Delta^{2}=-\frac{1}{3}(2 u-v)(u-v) .
$$

As to the homogeneous part of degree $4,(3.3), 3.4$ and 3.1 show that

$$
\Delta^{4}=\left(u K_{X}+v L\right)^{4}=8 F(u, v),
$$

where

$$
F(u, v)=56 u^{4}-160 u^{3} v+168 u^{2} v^{2}-77 u v^{3}+13 v^{4}
$$

hence $\frac{1}{24} \Delta^{4}=\frac{1}{3} F(u, v)$. Note that the polynomial $F$ can be rewritten as

$$
F(u, v)=28 u^{2}\left(2 u^{2}-3 u v+v^{2}\right)-v G(u, v)=28 u^{2}(2 u-v)(u-v)-v G(u, v),
$$

where $G(u, v)=76 u^{3}-140 u^{2} v+77 u v^{2}-13 v^{3} ;$ moreover, it is easy to see that

$$
\begin{aligned}
G(u, v) & =(2 u-v)\left(38 u^{2}-51 u v+13 v^{2}\right) \\
& =(2 u-v)(u-v)(38 u-13 v)
\end{aligned}
$$

Thus

$$
F(u, v)=(2 u-v)(u-v)\left[28 u^{2}-v(38 u-13 v)\right] .
$$

Therefore, the homogeneous part of degree 4 is

$$
\frac{1}{24} \Delta^{4}=\frac{1}{3}(2 u-v)(u-v)\left(28 u^{2}-38 u v+13 v^{2}\right) .
$$

In conclusion, putting all pieces together and collecting all common factors, we get (2.4).

\section{Second approach}

In this section, we obtain equation (2.4) again with another approach using Algorithm 3 in [7, Appendix]. To do that, it is more convenient to use coordinates $(x, y)=(1 / 2+u, v)$ in the plane of $\Gamma_{(X, L)}$. Let $S \subset \mathbb{P}^{3}$ be a smooth quadric surface not containing the point $w$ and consider the smooth threefold $V:=\pi^{-1}(S) \in\left|\pi^{*}\left(\mathcal{O}_{\mathbb{P}^{3}}(2)\right)\right|$. Clearly, $V \cap \pi^{-1}(w)=\emptyset$ and $\left(V, L_{V}\right)$ is a scroll over $S$ via $\left.\pi\right|_{V}: V \rightarrow S$, with $K_{V}+2 L_{V}=\left(\left.\pi\right|_{V}\right)^{*} \mathcal{O}_{\mathbb{P}^{3}}(4)_{S}$. Note also that

$$
V \in\left|K_{X}+2 L\right|
$$


in view of 2.3. According to the above quoted algorithm, consider the following exact sequence:

$$
0 \rightarrow x K_{X}+y L+(x-1) V \rightarrow x\left(K_{X}+V\right)+y L \rightarrow x K_{V}+y L_{V} \rightarrow 0,
$$

which by 4.1 can be rewritten as

$$
0 \rightarrow(2 x-1) K_{X}+(2(x-1)+y) L \rightarrow 2 x K_{X}+(2 x+y) L \rightarrow x K_{V}+y L_{V} \rightarrow 0
$$

The exact sequence 4.2 gives the following relation between $p_{(X, L)}$ and $p_{\left(V, L_{V}\right)}$ :

$$
p_{(X, L)}(2 x, 2 x+y)=p_{(X, L)}(2 x-1,2 x+y-2)+p_{\left(V, L_{V}\right)}(x, y)
$$

By 2, Theorem 6.1] we know that, in terms of coordinates $(a, b)$, the two polynomials can be written as

$$
p_{(X, L)}(a, b)=R_{(X, L)}(a, b) \cdot(2 a-b-1) \quad \text { and } \quad p_{\left(V, L_{V}\right)}(a, b)=R_{\left(V, L_{V}\right)}(a, b) \cdot(2 a-b-1)
$$

where $R_{(X, L)}$ and $R_{\left(V, L_{V}\right)}$ are polynomials in $(a, b)$ of degrees 3 and 2 , respectively. Thus (4.3) becomes

$$
R_{(X, L)}(2 x, 2 x+y)=R_{(X, L)}(2 x-1,2 x+y-2)+R_{\left(V, L_{V}\right)}(x, y) .
$$

The goal will be to find the explicit expression of the polynomial

$$
R_{(X, L)}(a, b):=A^{\prime} a^{3}+B^{\prime} a^{2} b+C^{\prime} a b^{2}+E^{\prime} b^{3}+F^{\prime} a^{2}+G^{\prime} a b+H^{\prime} b^{2}+J^{\prime} a+L^{\prime} b+M^{\prime}
$$

with rational coefficients, because $R_{\left(V, L_{V}\right)}$ is known by 7 , Theorem 4.3]. Actually, adapting the notation used there (see also [7, Example 4.2]) to our situation, we have

$$
S \cong \mathbb{P}^{1} \times \mathbb{P}^{1}, \quad A=\mathcal{O}_{\mathbb{P}^{3}}(4)_{S}, \quad K_{S}=\mathcal{O}_{\mathbb{P}^{3}}(-2)_{S}
$$

Hence $\chi\left(\mathcal{O}_{V}\right)=\chi\left(\mathcal{O}_{S}\right)=1, K_{S} \cdot A=-16$ and $A^{2}=32$. Moreover, from the exact sequence

$$
0 \rightarrow L-V=L+\pi^{*} \mathcal{O}_{\mathbb{P}^{3}}(-2) \rightarrow L \rightarrow L_{V} \rightarrow 0,
$$

we get $\chi\left(L_{V}\right)=\chi(L)-\chi\left(L+\pi^{*} \mathcal{O}_{\mathbb{P}^{3}}(-2)\right)$. Observe that

$$
\chi(L)=\chi(\mathcal{F})=2 \chi(H)=2 h^{0}\left(Y, \sigma^{*} \mathcal{O}_{\mathbb{P}^{3}}(3)-e\right)=38
$$

and

$$
\begin{aligned}
\chi\left(L+\pi^{*} \mathcal{O}_{\mathbb{P}^{3}}(-2)\right) & =\chi\left(\mathcal{F} \otimes \sigma^{*} \mathcal{O}_{\mathbb{P}^{3}}(-2)\right)=2 \chi\left(H \otimes \sigma^{*} \mathcal{O}_{\mathbb{P}^{3}}(-2)\right) \\
& =2 \chi\left(\sigma^{*} \mathcal{O}_{\mathbb{P}^{3}}(1)-e\right)=6 .
\end{aligned}
$$


Therefore, $\chi\left(L_{V}\right)=38-6=32$ and then from [7, Theorem 4.3] we deduce that

$$
R_{\left(V, L_{V}\right)}(x, y)=-4 x^{2}+12 x y-9 y^{2}+4 x-6 y-1
$$

Note that Serre duality on $X$ implies that $p_{(X, L)}(a, b)=p_{(X, L)}(1-a,-b)$, which in turn gives

$$
R_{(X, L)}(a, b)=-R_{(X, L)}(1-a,-b) .
$$

This leads by using MAPLE to the following relations:

$$
A^{\prime}=2 J^{\prime}+4 M^{\prime}, \quad B^{\prime}=-G^{\prime}, \quad C^{\prime}=-2 H^{\prime}, \quad F^{\prime}=-3 J^{\prime}-6 M^{\prime} .
$$

Using these relations, (4.6) and (4.5) with the pairs $(2 x, 2 x+y)$ and $(2 x-1,2 x+y-2)$ instead of $(a, b)$ to obtain the terms $R_{(X, L)}(2 x, 2 x+y)$ and $R_{(X, L)}(2 x-1,2 x+y-2)$, respectively, from (4.4) we deduce the following expressions for four further unknown coefficients:

$$
G^{\prime}=-12 E^{\prime}-30, \quad H^{\prime}=3 E^{\prime}+\frac{9}{2}, \quad J^{\prime}=-4 E^{\prime}-2 M^{\prime}-\frac{38}{3}, \quad L^{\prime}=2 E^{\prime}+M^{\prime}+\frac{9}{2} .
$$

Finally, by computing $p_{(X, L)}$ in $(0,0)$ and $(0,1)$, we get

$$
\begin{gathered}
1=p_{(X, L)}(0,0)=(-1) R_{(X, L)}(0,0)=-M^{\prime}, \\
38=\chi(L)=p_{(X, L)}(0,1)=(-2) R_{(X, L)}(0,1)=-12 E^{\prime}-14 .
\end{gathered}
$$

Hence $M^{\prime}=-1$ and $E^{\prime}=-13 / 3$. By replacing these values in the previous expressions of the coefficients, we deduce the final expression of $R_{(X, L)}$ in terms of the coordinates $(x, y)$ :

$$
R_{(X, L)}(x, y)=\frac{28}{3} x^{3}-22 x^{2} y+17 x y^{2}-\frac{13}{3} y^{3}-14 x^{2}+22 x y-\frac{17}{2} y^{2}+\frac{20}{3} x-\frac{31}{6} y-1,
$$

which leads to $p_{(X, L)}(1 / 2+u, v)$ as in 2.4$)$, keeping in mind that $p_{(X, L)}(x, y)=R_{(X, L)}(x, y)$ $\cdot(2 x-y-1)$ and $(x, y)=(1 / 2+u, v)$.

\section{A singular property of $\Gamma_{(X, L)}$}

Coming back to Question 2.1, we highlight an intriguing property of the Hilbert curve of our polarized fourfold $(X, L)$. As observed, we can regard $(X, L)$ as an adjunction theoretic scroll over $\mathbb{P}^{3}$ as well as a classical scroll over $Y$. Due to (2.3), since [2, Theorem 6.1] holds for scrolls of both types, the linear factor $(2 u-v)$ in 2.4 was a priori expected. The question is whether the residual degree 3 factor

$$
\phi(u, v)=\frac{1}{3}\left(28 u^{3}-66 u^{2} v+51 u v^{2}-13 v^{3}-u+v\right)
$$


defining a plane cubic $C$, is somehow related to the base threefold $\mathbb{P}^{3}$ ( $Y$, respectively) of our scroll $(X, L)$ for some polarization. Let us start with $\mathbb{P}^{3}$. By 2.1 with $x=u+1 / 2$ and $y=v$, we see that for any positive integer $a$ the canonical equation of the Hilbert curve of the polarized threefold $\left(\mathbb{P}^{3}, \mathcal{O}_{\mathbb{P}^{3}}(a)\right)$ is

$$
\frac{1}{6} \prod_{i=1}^{3}(-4 u+a v+i-2)=0
$$

and the same occurs for any positive $a \in \mathbb{Q}$. It is immediate to check that the polynomial on the left hand contains nontrivial homogeneous terms of degree 2, contrary to what happens for $\phi$. Therefore the cubic $C$ of equation $\phi(u, v)=0$ cannot be the Hilbert curve of $\left(\mathbb{P}^{3}, \mathcal{O}_{\mathbb{P}^{3}}(a)\right)$.

This shows that in general for an adjunction theoretic scroll, Question 2.1 has a negative answer.

Next consider $Y$. Any ample line bundle $M$ on $Y$ can be written as $M=a h-r e$ for suitable integers $a$ and $r$. For any divisor $D^{\prime}=\frac{1}{2} K_{Y}+\Delta^{\prime}$ on $Y$, the Riemann-Roch formula says that

$$
\chi\left(D^{\prime}\right)=\frac{1}{6} \Delta^{\prime 3}+\frac{1}{24}\left(2 c_{2}(Y)-K_{Y}^{2}\right) \cdot \Delta^{\prime}
$$

[1. p. 291]. Hence, letting $\Delta^{\prime}=u K_{Y}+v M$ and computing all required intersections, (5.1) leads to the canonical equation of the Hilbert curve of $(Y, M)$, which turns out to be

$$
\begin{aligned}
& p_{(Y, M)}\left(\frac{1}{2}+u, v\right) \\
= & \frac{1}{6}\left[-56 u^{3}+12(4 a-r) u^{2} v-6\left(2 a^{2}-r^{2}\right) u v^{2}+\left(a^{3}-r^{3}\right) v^{3}+2 u-(a-r) v\right] \\
= & 0 .
\end{aligned}
$$

This polynomial is proportional to $\phi(u, v)$ if and only if the matrix

$$
\left(\begin{array}{cccccc}
-56 & 12(4 a-r) & -6\left(2 a^{2}-r^{2}\right) & a^{3}-r^{3} & 2 & r-a \\
28 & -66 & 51 & -13 & -1 & 1
\end{array}\right)
$$

has rank 1. An immediate check shows that this happens if and only if $(a, r)=(3,1)$, i.e., for $M=H$. We thus see that

$$
\phi(u, v)=-p_{(Y, H)}\left(\frac{1}{2}+u, v\right) .
$$

Therefore, the factor $\phi$ defines the Hilbert curve of the base $Y$ of our classical scroll $(X, L)$, endowed with the average polarization $H=\frac{1}{2} \operatorname{det} \mathcal{F}$ induced by the ample vector bundle $\mathcal{F}$. 
Moreover, we see that, in the special situation we are dealing with, Question 2.1 has a positive answer regarding $(X, L)$ as a classical scroll over $Y$, while this is not the case when we look at it as an adjunction theoretic scroll over $\mathbb{P}^{3}$.

Remark 5.1. The conclusion concerning $(X, L)$ as a scroll over $\mathbb{P}^{3}$ can be obtained more geometrically, arguing as follows. The Hilbert curve of $\left(\mathbb{P}^{3}, \mathcal{O}_{\mathbb{P}^{3}}(a)\right)$ consists of three parallel evenly spaced lines, while, from the real point of view, the cubic $C$ consists of the line $u-v=0$ plus an ellipse: actually, a straightforward verification shows that the conic $\gamma$ of equation $28 u^{2}-38 u v+13 v^{2}-1=0$ is an ellipse whose axes, determined by the eigenvectors of the matrix

$$
A_{\infty}:=\left(\begin{array}{cc}
28 & -19 \\
-19 & 13
\end{array}\right)
$$

are $3 u-2 v=0$ and $2 u+3 v=0$. From another perspective, removing both linear factors $2 u-v$ and $u-v$ from (2.4) one could ask whether the conic $\gamma$ described by the residual degree 2 polynomial is the Hilbert curve of some polarized or $\mathbb{Q}$-polarized surface $(S, \mathcal{L})$. Even in this case the answer is negative. Otherwise, taking into account that the canonical equation of the Hilbert curve of $(S, \mathcal{L})$ is

$$
\frac{1}{2}\left(K_{S}^{2} u^{2}+2 K_{S} \cdot \mathcal{L} u v+\mathcal{L}^{2} v^{2}+\left(2 \chi\left(\mathcal{O}_{S}\right)-\frac{1}{4} K_{S}^{2}\right)\right)=0
$$

(5.2) would imply the existence of a nonzero rational number $\rho$ such that

$$
K_{S}^{2} \mathcal{L}^{2}-\left(K_{S} \cdot \mathcal{L}\right)^{2}=\rho^{2} \operatorname{det} A_{\infty}=3 \rho^{2}>0
$$

but this contradicts the Hodge index theorem.

\section{Acknowledgments}

The first author is a member of G.N.S.A.G.A. of the Italian INdAM. He would like to thank the PRIN 2015 Geometry of Algebraic Varieties and the University of Milano for partial support. During the preparation of this paper, the second author was partially supported by the National Project Anillo ACT 1415 PIA CONICYT and the Proyecto VRID N.214.013.039-1.OIN of the University of Concepción. The authors are grateful to the referee for useful remarks.

\section{References}

[1] M. C. Beltrametti, A. Lanteri and M. Lavaggi, Hilbert surfaces of bipolarized varieties, Rev. Roumaine Math. Pures Appl. 60 (2015), no. 3, 281-319. 
[2] M. C. Beltrametti, A. Lanteri and A. J. Sommese, Hilbert curves of polarized varieties, J. Pure Appl. Algebra 214 (2010), no. 4, 461-479.

[3] M. C. Beltrametti and A. J. Sommese, Comparing the classical and the adjunctiontheoretic definition of scrolls, in: Geometry of Complex Projective Varieties, 55-74, Sem. Conf. 9, Mediterranean, Rende, 1993.

[4] - The Adjunction Theory of Complex Projective Varieties, De Gruyter Expositions in Mathematics 16, Walter de Gruyter \& Co., Berlin, 1995.

[5] P. Griffiths and J. Harris, Principles of Algebraic Geometry, Pure and Applied Mathematics, Wiley-Interscience, New York, 1978.

[6] A. Lanteri, Characterizing scrolls via the Hilbert curve, Internat. J. Math. 25 (2014), no. $11,1450101,17$ pp.

[7] A. Lanteri and A. L. Tironi, Hilbert curve characterizations of some relevant polarized manifolds, arXiv:1803.01131.

[8] A. L. Tironi, Scrolls over four dimensional varieties, Adv. Geom. 10 (2010), no. 1, $145-159$.

[9] _ Nefness of adjoint bundles for ample vector bundles of corank 3, Math. Nachr. 286 (2013), no. 14-15, 1548-1570.

Antonio Lanteri

Dipartimento di Matematica "F. Enriques", Università degli Studi di Milano, Via C.

Saldini, 50, I-20133 Milano, Italy

E-mail address: antonio.lanteri@unimi.it

Andrea Luigi Tironi

Departamento de Matemática, Universidad de Concepción, Casilla 160-C, Concepción, Chile

E-mail address: atironi@udec.cl 